\title{
Development of an Efficient Methodology for the Mapping and Digital Analysis of 3D Surfaces via SEM
}

\author{
D. Stalla ${ }^{1}$, K. Banks ${ }^{2}$, J. Brown ${ }^{3}$, F. Bunyak ${ }^{4}$, E. Giuliano ${ }^{2}$, T. White ${ }^{1}$ \\ ${ }^{1 .}$ Electron Microscopy Core, University of Missouri, Columbia, MO, USA \\ 2. College of Veterinary Medicine, University of Missouri, Columbia, MO, USA \\ ${ }^{3 .}$ Dept. of Mechanical and Aerospace Engineering, University of Missouri, Columbia, MO, USA \\ 4. Department of Computer Science, University of Missouri, Columbia, MO, USA
}

Performing efficient, reproducible measurements of a 3-dimensional surface poses a nontrivial challenge in SEM imaging. This process is made significantly more difficult when considering a sphere, a configuration devoid of any easily identifiable reference points.

For this work, we have been tasked with investigating changes in morphology of diamond-embedded ophthalmic debridement burrs (specifically, the $3.5 \mathrm{~mm}$ Pterygium Burr and Chuck used with an Algerbrush II) before and after clinical use to test the efficacy of various cleaning treatments. The specific structural modifications that will be evaluated are comprised of:

1. Topographical erosion of individual implanted diamond particles via SE

2. Modification of impregnation sites via BSE

3. Residual contamination via BSE

Computer-aided image processing will be instrumental in the identification and classification of the various morphological deviations: backscatter electron images will be used to generate a background mask to separate the implanted diamond from the underlying $3.5 \mathrm{~mm}$ tip surface, while geodesic active contours will define and monitor fine topographical evolution within individual diamond particles.

A custom holder, compatible with our existing stage, was designed and machined. The tip chuck sits in the holder at a user-prescribed orientation; utilization of the stage tilt, parallel to the arm's positioning, thus readily allows for iterative scans down a given 'longitude' of the tip at well-defined latitudes. However, as the holder maintains the tip off-axis, a full 'brute force' mapping of the sphere demands a prohibitive amount of work: each change to a new longitude requires venting the chamber, manual reorientation within the holder, reestablishing vacuum, and realigning under the beam. In addition to being considerably time inefficient, this method is additionally non-optimal as it carries difficulties in replicating, upon post-treatment analysis, the exact regions from initial imaging.

In addressing this, implicit vector analysis allows for the determination of the precise stage tilt $(\alpha)$, stage rotation $(\beta)$, and scan rotation $(\delta)$ required to achieve consistent imaging at a given latitude $(\phi)$ and longitude $(\lambda)$ (Figure 2 ). In addition to greatly expediting the imaging process by yielding a dramatically wider range of simultaneously accessible regions (we're able to concurrently image a third of the tip from each orientation within the holder, positions corresponding to $-60^{\circ} \leq \lambda \leq 60^{\circ}$ and $0 \leq \phi \leq 90^{\circ}$ ), this allows for the calculation and selection of positions that are optimal both in number and spatial distribution. 

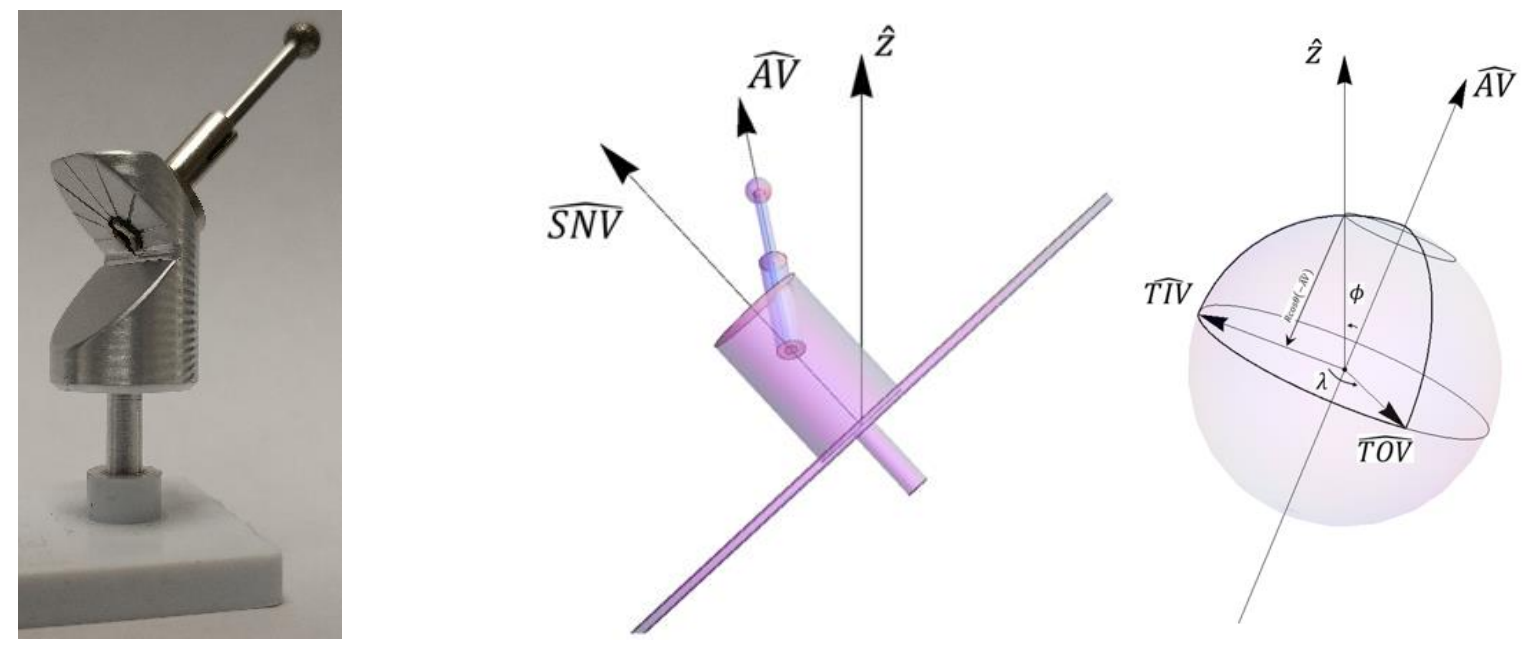

Figure 1. (left) Custom built $3.5 \mathrm{~mm}$ burr holder, with burr chuck installed; angle markers $\left(30^{\circ}\right.$ increments) are visible on underside of holder. (middle/right) Schematic diagrams of the defined vectors considered for geometric analysis. The position and orientation of the imaged region of the tip $(\phi, \lambda)$ relative to the beam upon arbitrary stage tilt and rotation $(\alpha, \beta)$ can be expressed via application of vector rotations about the $\mathrm{y}$-axis and stage normal vector, respectively:

$$
\begin{aligned}
& \cos \alpha_{\text {req }}(\phi, \lambda)=\frac{\cos \phi_{\text {desired }}+\sin \phi_{\text {desired }} \cos \lambda_{\text {desired }}}{\sqrt{2}} \\
& \cos \beta_{\text {req }}(\phi, \lambda)=\frac{\cos \phi_{\text {desired }}-\sin \phi_{\text {desired }} \cos \lambda_{\text {desired }}}{\sqrt{2} \sin \alpha_{\text {req }}} \\
& \cos \delta_{\text {req }}(\phi, \lambda)=\frac{\sin \phi_{\text {desired }}-\cos \phi_{\text {desired }} \cos \lambda_{\text {desired }}}{\sqrt{2} \sin \alpha_{\text {req }}}
\end{aligned}
$$

Figure 2. Analytically determined stage tilt $(\alpha)$, stage rotation $(\beta)$, and scan rotation $(\delta)$ required to image a specific desired latitude $(\phi)$ and longitude $(\lambda)$ on the burr tip.
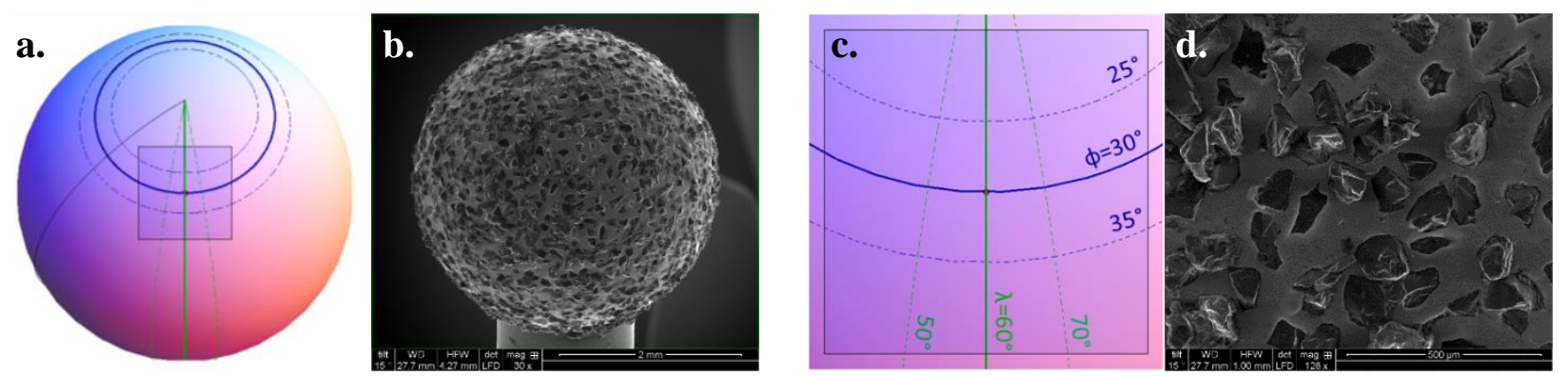

Figure 3. Modeled representation $(a / c)$, and corresponding SE image $(b / d)$, of the point corresponding to $30^{\circ}$ latitude and $60^{\circ}$ longitude at HFWs of $\sim 4.3 \mathrm{~mm}(a / b)$ and $1.0 \mathrm{~mm}(\mathrm{c} / d)$. 